\title{
PREPARAÇÃo E CARACTERIZAÇÃo DE BLENDAS DE QUITOSANA/POLI(ÁLCOOL VINÍLICO) RETICULADAS QUIMICAMENTE COM GLUTARALDEÍDO PARA APLICAÇÃO EM ENGENHARIA DE TECIDO
}

\author{
Ezequiel de Souza Costa Jr. \\ Coordenação do Curso Técnico de Mecânica, Centro Federal de Educação Tecnológica - MG, Av. Amazonas, 5253, \\ 30480-000 Belo Horizonte - MG, Brasil \\ Herman Sander Mansur* \\ Departamento de Engenharia Metalúrgica e de Materiais, Universidade Federal de Minas Gerais, Rua Espírito Santo, 35 , \\ 30160-030 Belo Horizonte - MG, Brasil
}

Recebido em 3/10/07; aceito em 14/3/08; publicado na web em 1/9/08

\begin{abstract}
PREPARATION AND CHARACTERIZATION OF CHITOSAN/POLY(VINYL ALCOHOL)BLEND CHEMICALLY CROSSLINKED BY GLUTARALDEHYDE FOR TISSUE ENGINEERING APPLICATION. In this study, novel Chitosan/PVA based films were chemically crosslinked by glutaraldehyde, under $\mathrm{pH}=(4,00 \pm 0,05)$, in order to achieve structures tailored for wound tissue engineering applications. Both precursors and developed films were characterized by FTIR, SEM and XRD in order to determine the presence of chemicals groups and nanostructural order, respectively. The results have shown that the glutaraldehyde crosslinking have altered the crystallinity of pure chitosan and the increase on the $\mathrm{C}=\mathrm{N}$ bands and simultaneous decrease on $\mathrm{NH}_{2}$ bands suggested that Chitosan/GA crosslinking has preference to occur in carbon-2 of the saccharide ring by the Schiff's base reaction. Also, FTIR spectroscopy clearly showed that crosslinking has also taken place with blends of PVA and chitosan. The mechanical properties presented high degree dependence with on the increase of the content of chitosan and glutaraldehyde. The results have indicated that, by controlling the ratio [PVA]/[chitosan] in the blends and the extent of chemical crosslinking, it was possible to tailor the hybrid network produced aiming to obtain properties of interest for the specific application.
\end{abstract}

Keywords: chitosan; poly(vinyl alcohol); crosslinking.

\section{INTRODUÇÃO}

Num sentido amplo a engenharia de tecidos visa fabricar partes de órgãos e tecidos de organismos vivos para sua substituição. Esse ramo do conhecimento tem se desenvolvido devido à crescente demanda por órgãos e tecidos em função de acidentes e/ou tratamentos de enfermidades diversas. ${ }^{1}$ De forma a suprir essa necessidade, as blendas de polímeros naturais e sintéticos têm se mostrado uma alternativa viável, pois é possível trabalhar diversas propriedades a fim de viabilizar as características específicas de cada aplicação.

A quitosana (QUI) é um polímero obtido principalmente a partir da desacetilação alcalina da quitina, a qual é o segundo polímero natural mais abundante depois da celulose e é encontrado nos exoesqueletos de artrópodes e alguns fungos. Ela tem propriedades interessantes para aplicação em engenharia de tecido, tais como biocompatibilidade, biodegradabilidade, acelera a recuperação de lesões, reduz o nível do colesterol sanguíneo, estimula os efeitos do sistema imunológico, além de ter se mostrado um composto bacteriostático e fungistático, ${ }^{2}$ bem como os resultados de sua degradação em organismos vivos que são as glicosaminas, substâncias não tóxicas. Uma das características mais importantes deste polissacarídeo é o seu grau de desacetilação (GD) que representa a proporção de resíduos acetilados ainda presentes no mesmo, após o processamento para sua obtenção. A quitosana se degrada pela hidrólise enzimática e apresenta cinética de degradação aparentemente relacionada ao grau de cristalinidade que é controlado principalmente pelo GD, ${ }^{3}$ entretanto sua resistência mecânica e sua maleabilidade são limitadas principalmente para aplicação como filmes em bandagens, por exemplo, e por isso a mistura

*e-mail: hmansur@demet.ufmg.br da mesma com outros polímeros é um procedimento utilizado para alterar ou obter as propriedades de interesse. O grande desafio consiste em obter um material que resista de forma controlada à degradação em ambiente fisiológico. Com o objetivo de melhorar as propriedades mecânicas e variar a taxa de degradação deste polissacarídeo utiliza-se a mistura da mesma com outros hidrogéis poliméricos sintéticos, tais como o poli(álcool vinílico) (PVA), bem como com reticulantes, que permitem a formação de ligações cruzadas com a cadeia polimérica principal, favorecendo a obtenção de redes, além de bloquear os grupos amino com um agente bifuncional.

O PVA é um polímero produzido pela polimerização do acetato de vinila seguida de reação de hidrólise do poli(acetato de vinila) em poli(álcool vinílico). A relação entre o percentual de hidroxilas no copolímero final, após a reação de hidrólise, e o número total inicial de radicais ácido acético representa o grau de hidrólise (GH) do PVA. Esse parâmetro é importante na caracterização das propriedades deste polímero. Um aumento do GH implica em redução da solubilidade na água, associada à estabilização energética promovida pelas ligações de hidrogênio intra e intercadeias poliméricas, e no aumento da adesão em superfícies hidrofílicas, da viscosidade e da resistência à tração. ${ }^{4}$ O hidrogel de PVA possui excelente transparência, consistência macia quando na forma de membrana, além de apresentar excelente resistência química e ser biocompatível. ${ }^{5}$

Reticulantes são moléculas de peso molecular muito menor que o peso molecular da cadeia principal entre duas ligações cruzadas consecutivas e normalmente apresentam, no mínimo, dois grupos funcionais reativos que permitam a formação de ponte entre cadeias poliméricas. ${ }^{2,6}$ Os hidrogéis reticulados são cadeias poliméricas interconectadas pelo reticulante conduzindo a uma formação de rede tridimensional. As propriedades dos hidrogéis reticulados dependem 
principalmente da sua densidade de ligações cruzadas, notadamente da relação de moles do agente reticulante com os da unidade de repetição do polímero. Exige-se um número crítico de ligações cruzadas por cadeia para permitir a formação da rede.

O glutaraldeído (GA) é um agente reticulante normalmente usado na formação de redes de polipeptídios e proteínas devido à reatividade dos grupos aldeídos, que prontamente formam bases de Schiff com os grupos amino das proteínas. É também usado como agente reticulante do PVA e alguns polissacarídeos, tais como a heparina, o ácido hialurônico e a quitosana., 7 A reticulação da quitosana com GA a torna mais resistente do ponto de vista físico, químico e microbiológico. ${ }^{8}$

O objetivo principal deste trabalho consistiu na preparação e caracterização de diversas proporções de misturas de QUI/PVA, com reticulação química por GA para aplicação potencial em engenharia de tecidos para obtenção de uma matriz dérmica.

\section{PARTE EXPERIMENTAL}

\section{Materiais}

Todos os sais e reagentes foram utilizados em grau analítico e água Milli-Q foi utilizada em todas as soluções (18,0 M $\Omega$ ).

Uma solução de 1,0\% (em massa) de quitosana (Sigma-Aldrich, $\mathrm{GD}=75,6 \%$, viscosidade $=1406 \mathrm{cP}-1,0 \%$ em $1 \%$ de ácido acético) foi preparada pela dissolução de $2,5 \mathrm{~g}$ em $250 \mathrm{~mL}$ de solução de $\mathrm{CH}_{3} \mathrm{COOH}$ (Sigma) 2\% (em massa). Para a solubilização e homogeneização, a solução foi agitada magneticamente por um período entre $72-120 \mathrm{~h}$.

Amostras de PVA (GH=80\% e massa molar(MM) de 9000 a 10000Da, Sigma-Aldrich). As informações relativas ao GH e MM foram obtidas do relatório de especificações técnicas do fornecedor.

Glutaraldeído (GA) ou 1,5 pentanodial (Sigma-Aldrich), agente reticulante (solução aquosa $25 \%$ ).

\section{Preparação dos filmes}

O hidrogel de PVA foi preparado pela dissolução de 5,0 g de pó do polímero em $100 \mathrm{~mL}$ de água Milli-Q, sob agitação magnética à temperatura de $70 \pm 2{ }^{\circ} \mathrm{C}$. Após a solução ter sido resfriada na temperatura ambiente, o $\mathrm{pH}$ foi corrigido para 2,00 $\pm 0,05 \mathrm{com} \mathrm{HCl}$ $1,0 \mathrm{M}$ (Sigma).

As soluções de misturas de QUI/PVA nas proporções de 25/75; 50/50 e 75/25 (em massa), bem como de quitosana e PVA puros em pH 4,0 $\pm 0,05$, ajustado pela adição de $\mathrm{NaOH}$ (Carlo Erba), foram vertidas em placas plásticas e secas a temperatura ambiente por 72 $\mathrm{h}$, conforme indicado na Tabela 1.

A reticulação foi realizada pela adição em cada proporção de mistura de 1,0 e 5,0\% de GA (em massa de polímero).

\section{Avaliação qualitativa}

As observações visuais qualitativas foram realizadas levandose em conta a solubilidade, miscibilidade e segregação de fase nas blendas, alem de avaliar a espessura dos filmes obtidos com um micrometro Mitotoyo $( \pm 10 \mu \mathrm{m})$.

\section{Microscopia eletrônica de varredura}

As morfologias dos filmes obtidos foram avaliadas através de microscopia eletrônica de varredura (MEV) (JSM 6360LV,JEOL/Noran) acoplada a espectrômetro de energia dispersiva (EDS). As imagens foram obtidas utilizando tensão de aceleração de 10 e 20 kV. Antes
Tabela 1. Identificação e proporção das blendas

\begin{tabular}{lccc}
\hline $\mathrm{CP}$ & Quitosana GD=75,6\% & PVA GH=80\% & GA $(\%)$ \\
\hline 1 & 0 & 1 & 0 \\
2 & 1 & 0 & 0 \\
3 & 1 & 3 & 0 \\
4 & 1 & 1 & 0 \\
5 & 3 & 1 & 0 \\
6 & 0 & 1 & 1 \\
7 & 1 & 0 & 1 \\
8 & 1 & 3 & 1 \\
9 & 1 & 1 & 1 \\
10 & 3 & 1 & 1 \\
11 & 0 & 1 & 5 \\
12 & 1 & 0 & 5 \\
13 & 1 & 3 & 5 \\
14 & 1 & 1 & 5 \\
15 & 3 & 1 & 5 \\
\hline
\end{tabular}

do exame as amostras foram cobertas com fina camada de ouro por aspersão usando baixa taxa de deposição, refrigeradas e colocadas à máxima distância do alvo a fim de evitar danos às mesmas.

\section{Espectroscopia de infravermelho}

Com o objetivo de caracterizar a presença de grupos químicos específicos nos materiais e filmes obtidos foi realizada análise de infravermelho (IV) usando os modos de transmitância e ATR (reflexão total atenuada). Os espectros foram obtidos na faixa de número de onda de 4000 a $650 \mathrm{~cm}^{-1}$ durante 64 varreduras, com resolução de $2 \mathrm{~cm}^{-1}$ (Paragon 1000, Perkin-Elmer, USA). Os espectros de IV foram normalizados e as bandas de vibração foram associadas aos principais grupos químicos.

\section{Titulação potenciométrica}

A determinação do grau de desacetilação da quitosana foi realizada através de titulação potenciométrica: $0,2 \mathrm{~g}$ de quitosana foi dissolvida em $20 \mathrm{~mL}$ de solução padronizada de $\mathrm{HCl} 0,10 \mathrm{~N}$ e diluída com $10 \mathrm{~mL}$ de água destilada. Em seguida, sob agitação constante titulou-se a solução com $\mathrm{NaOH}$ 0,01M padronizado, obtendo-se a curva típica de titulação potenciométrica.

\section{Difração de raios-X}

Padrão de difração de raios-X (DRX) foi obtido das matériasprimas e dos filmes produzidos (Philips, PW1710) usando a radiação $\mathrm{K} \alpha$ do cobre com $\lambda=1,54056 \AA$. A análise de DRX foi conduzida com $2 \theta$ variando de 3,01 a $90,00^{\circ}$ com passo de $0,06^{\circ}$. O padrão de difração do PVA foi analisado com base na estrutura monoclínica para a célula unitária ( $a=7,81 \AA$; $b=2,52 \AA$; $c=5,51 \AA$ e $\beta=91^{\circ} 42^{\prime}$ ). ${ }^{9-11}$ A quitosana foi avaliada segundo a estrutura ortorrômbica $(a=8,9 \AA, b=10,25 \AA$ e $\mathrm{c}=17,0 \AA$ ) e monoclínica com $\beta=88^{\circ}$. $^{12-14}$

\section{Propriedades mecânicas}

Os testes de tração realizados para avaliar o comportamento mecânico (tensão máxima de tração $(\sigma)$, deformação específica (DE) e módulo de elasticidade(E)) dos filmes obtidos foram realizados em equipamento Emic DL 3000, utilizando uma célula de carga de 50N, velocidade de ensaio de $5 \mathrm{~mm} / \mathrm{min}$ e corpos de prova na forma de 
gravata borboleta conforme ASTM D882/97, ${ }^{15}$ temperatura de ensaio de $21 \pm 2{ }^{\circ} \mathrm{C}$ e umidade relativa de $52 \pm 8 \%$.

A tensão de tração foi calculada pela Equação 1 onde (F) representa a força de tração e (A) a área da secção transversal da amostra ensaiada.

$$
\sigma=\frac{\mathrm{F}}{\mathrm{A}}
$$

A Equação 2 apresenta a obtenção da deformação específica (DE) onde $(\Delta \mathrm{l})$ é a variação de comprimento e $\left(1_{0}\right)$ é o comprimento inicial do corpo de prova ensaiado.

$\mathrm{DE}=\frac{\Delta \mathrm{l}}{\mathrm{l}_{0}} \times 100$

O módulo de elasticidade (E) foi obtido conforme a Equação 3 onde $\varepsilon$ representa a deformação unitária.

$$
\mathrm{E}=\frac{\Delta \sigma}{\Delta \varepsilon}
$$

\section{RESULTADOS E DISCUSSÃO}

\section{Avaliação qualitativa}

Os filmes apresentaram-se com espessura de $75 \pm 25 \mu \mathrm{m}$ e nenhum desvio foi observado no que diz respeito à solubilidade, miscibilidade e segregação de fases ao avaliar as proporções de QUI/PVA, ou seja, pode-se afirmar que o sistema formou uma mistura uniforme. No que diz respeito à coloração, flexibilidade e ao aspecto visual a Tabela 2 apresenta a avaliação qualitativa das características observadas nos filmes obtidos de quitosana, PVA e blendas QUI/PVA sem reticulação e reticulados com 1,0 e 5,0\% de GA.

\section{Microscopia eletrônica de varredura (MEV)}

Os filmes puros e blendas analisados por MEV não apresentaram alterações, heterogeneidades ou segregações detectáveis na superfície ou na secção transversal. Somente na amostra de blenda do CP $3(\mathrm{Q} / \mathrm{P} /$ GA-1:3-1\%) foram observadas algumas marcas escuras sugerindo a presença de vazios que surgiram durante o processo de secagem, provavelmente devido à separação de fase conforme mencionado por, Don et al. ${ }^{16} \mathrm{em}$ função do teor da proporção QUI/PVA.

Tabela 2. Avaliação qualitativa dos filmes moldados

\begin{tabular}{lcc}
\hline CP & Observações & Esp. $(\mu \mathrm{m}) \pm 10 \%$ \\
\hline 1 & Transparente, flexível & 50 \\
2 & Esbranquiçado, flexível, segregado & 110 \\
3 & Transparente, flexível & 110 \\
4 & Transparente, flexível, segregado & 75 \\
5 & Transparente, flexível, pouco segregado & 65 \\
6 & Transparente, flexível & 50 \\
7 & Amarelo claro, segregado & 80 \\
8 & Amarelo claro, pouco segregado & 80 \\
9 & Amarelo claro, segregado & 90 \\
10 & Amarelo claro, pouco segregado & 90 \\
11 & Segregado, flexível & 50 \\
12 & Amarelo escuro, segregado & 95 \\
13 & Amarelo escuro, segregado & 75 \\
14 & Amarelo escuro, segregado & 80 \\
15 & Amarelo escuro, segregado & 110 \\
\hline
\end{tabular}

\section{Espectroscopia de infravermelho}

A espectroscopia de IV foi usada para avaliar os grupos químicos dos polímeros (quitosana e PVA), bem como a reticulação dos filmes com glutaraldeído.

A Tabela 3 apresenta as principais bandas de IV e grupos químicos usualmente associados à quitosana e ao copolímero PVA/PVAc.

Brugnerotto e colaboradore ${ }^{17}$ apresentaram um resumo sobre os métodos utilizados recentemente para avaliar o grau de desacetilação de quitina e quitosana onde se verifica a utilização das bandas de referência como sendo as de 3450; 2878; 1430; 1070 e, $1030 \mathrm{~cm}^{-1} \mathrm{e}$ as bandas características como as de $1655 ; 1630 \mathrm{e}$ $1560 \mathrm{~cm}^{-1}$, além de sugerir que, para qualquer GD de quitina ou quitosana, as bandas de 1420 e $1320 \mathrm{~cm}^{-1}$, como de referência e característica são mais estáveis independente da técnica, estado ou estrutura secundária.

A partir da Equação 4 e utilizando o valores de absorbância das bandas obtém-se o GD de $80 \pm 5 \% \quad(n=3)$ que corresponde a um desvio de $7 \%$ em relação ao valor fornecido pelo fabricante que é de $75,6 \%$, evidenciando razoável aproximação.

$$
\frac{A_{1320}}{A_{1420}}=0,3822+0,03133 \times(100-G D)
$$

A Figura 1 apresenta o espectro de infravermelho das blendas obtidas de QUI/PVA sem reticulação, conforme as proporções da Tabela 1 , bem como dos componentes puros. Observa-se um alargamento na banda de 3200 a $3600 \mathrm{~cm}^{-1}$ normalmente atribuída ao estiramento do $\mathrm{OH}$, e do $-\mathrm{NH}_{2}$. Com o aumento do teor de quitosana na blenda também se observa um aumento na banda de $1730 \mathrm{~cm}^{-1}$ normalmente atribuída ao estiramento da amida $(\mathrm{C}=\mathrm{O})$, bem como a banda de $1250 \mathrm{~cm}^{-1}$ que pode ser atribuída à deformação do $-\mathrm{NH}_{3}^{+}$, estiramento do $\mathrm{CO}$ e deformação do $\mathrm{OH}$ ocorrida devido à mistura dos polímeros. ${ }^{18,19}$

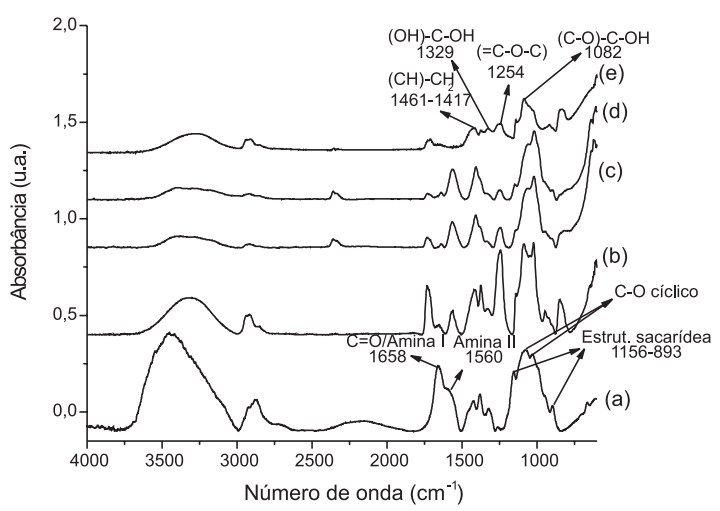

Figura 1. Espectros de IV dos filmes de quitosana pura (a) e blenda $Q / P(3: 1)$ (b); $Q / P(1: 1)$ (c); $Q / P(1: 3)(d)$; e PVA puro (e)

A Figura 2 apresenta o grupo de espectros dos filmes de quitosana reticulados. A reticulação química da quitosana com o GA ocorre a partir do nitrogênio nucleofílico do grupo amino $\left(-\mathrm{NH}_{2}\right)$ que reage com o carbono do aldeído, o qual desloca o oxigênio do aldeído e resulta na perda da molécula de água formando assim a ligação $\mathrm{C}=\mathrm{N}(\sim 1648$ $\left.\mathrm{cm}^{-1}\right)$, base de Shiff. ${ }^{20}$ Quando o $\mathrm{pH}$ do ambiente da reação é neutro ou alcalino ele facilita a formação do $-\mathrm{NH}_{2}\left(\sim 1558 \mathrm{~cm}^{-1}\right)$ da quitosana $(\mathrm{pKa}=6,3)$, que é essencial para a formação da base de Shiff . Em ambiente ácido, entretanto, $-\mathrm{NH}_{3}^{+}\left(1548 \mathrm{~cm}^{-1}\right)$ tem mais possibilidade de se formar, pois ele reduz significativamente a nucleofilicidade do nitrogênio, diminuindo a atividade da reação da base de Shiff. ${ }^{18,19}$ 
Tabela 3. Relação de bandas de FTIR e grupos químicos da quitosana ${ }^{17,18}$ e do copolímero PVA/PVAc ${ }^{4}$

\begin{tabular}{|c|c|c|c|c|c|}
\hline Material & Bandas $\left(\mathrm{cm}^{-1}\right)$ & Grupos & Material & Bandas $\left(\mathrm{cm}^{-1}\right)$ & Grupos \\
\hline \multirow{8}{*}{ QUITOSANA } & $3570-32003450$ & $v \mathrm{OH}$ ligado $v \mathrm{NH}_{2}$ & \multirow{8}{*}{ QUITOSANA } & 1321 & vC-N (primário) \\
\hline & $2955-28452922$ & vC-H (assimétrico) & & 1260 & vC-N (secundário) \\
\hline & 2878 & vC-H (simétrico) & & 1154 e 896 & vCOC (glicose $-\beta-1-4)$ \\
\hline & $1900-15001658$ & Amida $\mathrm{I} v \mathrm{C}=\mathrm{O}$ & & 11601154 & Hidrogênio ligado \\
\hline & $1650-15501658-1630$ & $\delta \mathrm{N}-\mathrm{H}(\mathrm{I})$ & & $1300-1000$ & vC-O (cíclico) \\
\hline & $1570-15151560$ & $\delta \mathrm{N}-\mathrm{H}(\mathrm{II})$ & & 897 & vC-O (cíclico) \\
\hline & 14651423 & $\delta \mathrm{OH} \mathrm{e} \mathrm{CH}_{2}$ (tesoura) & & $1640-1690$ & $v \mathrm{C}=\mathrm{NO}$ (fraca) (Base Schiff) \\
\hline & $1340-12501379$ & \&C-N (terciária) & & & \\
\hline PVA e PVAc & $3550-3200$ & $v(\mathrm{OH}) \mathrm{OH} \ldots \mathrm{OH}$ & PVA & 1329 & $\delta(\mathrm{OH})-\mathrm{C}-\mathrm{OH}$ \\
\hline PVA e PVAc & $2937-2870$ & $v(\mathrm{CH})$ & PVA & 1254 & $v_{\mathrm{ss}}(=\mathrm{CO}-\mathrm{C})$ \\
\hline PVAc & $1727-1712$ & $v(\mathrm{C}=\mathrm{O})$ & PVA & 1084 & $v(\mathrm{C}-\mathrm{O})-\mathrm{C}-\mathrm{OH}$ \\
\hline PVA e PVAc & $1650-1630$ & $\delta(\mathrm{OH}) \mathrm{OH} . . . \mathrm{OH}$ & PVA e PVAc & 922 & $\delta(\mathrm{CH})-\mathrm{CH}_{2}$ \\
\hline PVA & $1461-1417$ & $\delta(\mathrm{CH})-\mathrm{CH}_{2}$ & PVA & 842 & $v(C-C)$ \\
\hline PVA & 1366 & $\delta \mathrm{CH}-\mathrm{R}-\mathrm{CH}_{3}$ & & & \\
\hline
\end{tabular}

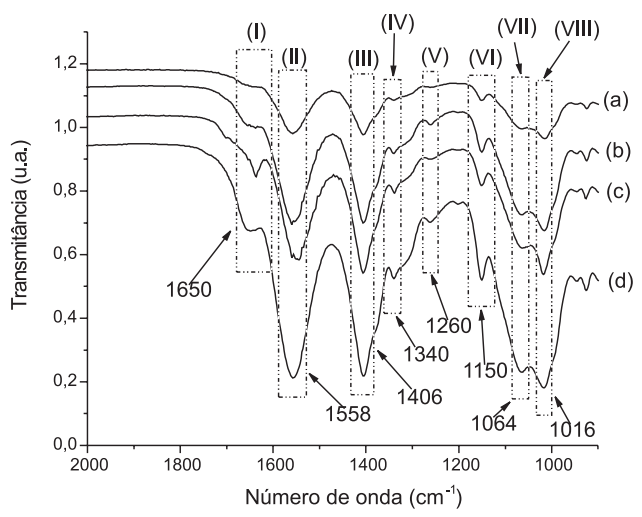

Figura 2. Espectros de IV dos filmes de quitosana pura (a) e reticulados com $0,5 \%$ (b); 1,0\% (c) e 5,0\% (d) de GA

Semelhante ao obtido por Monteiro Jr. ${ }^{21}$ e Wang e colaboradores ${ }^{18}$ comparando os espectros de FTIR, primeiramente observa-se a banda de $1648 \mathrm{~cm}^{-1}$, atribuída à ligação imina $(\mathrm{C}=\mathrm{N})$, e a segunda em $1558 \mathrm{~cm}^{-1}$ associada à ligação amina $\left(-\mathrm{NH}_{2}\right)$. Na região de 1720 $\mathrm{cm}^{-1}$, relacionada aos grupos aldeídos livres (-COH), não se observa variação com o aumento do teor de GA o que sugere que todo o GA foi ligado à quitosana, possivelmente, em função relação de grupos aminas disponíveis na quitosana e do grupos carbonila do GA.

$\mathrm{O}$ aumento do teor de GA também causa um aumento na intensidade da frequiência de $1562 \mathrm{~cm}^{-1}$ associada às ligações etilênicas; o mesmo comportamento foi observado na freqüência de $2922 \mathrm{~cm}^{-1}$ relativa ao estiramento dos grupos $\mathrm{C}$-H. Este fato pode ser atribuído ao aumento das contribuições da molécula de GA na reação QUIGA que promove o aumento na reticulação da cadeia. O aumento da intensidade das bandas relativas à ligação imina também sugere que a reticulação do GA ocorre preferencialmente via base de Shiff no carbono 2 do anel glicossídico, ${ }^{22}$ em detrimento da ligação com os grupos hidroxilas nos carbonos 3 e 6 .

A partir do espectro de FTIR relativo às blendas nas proporções de $25 \%$ de quitosana e de $75 \%$ PVA (em massa de polímero) onde se observou os picos das bandas de 1110, 1650 e $1638 \mathrm{~cm}^{-1}$ associadas respectivamente à reticulação do PVA e à formação do grupo imina a partir da amina da quitosana durante a reticulação pelo GA, ${ }^{18,19}$ construiu-se a Figura 3 que apresenta a evolução da formação de iminas e a redução das aminas, devido à reticulação da quitosana pelo GA e a Figura 4 que apresenta a evolução da banda de 1110 $\mathrm{cm}^{-1}$ relativa à reticulação do PVA pelo GA.
Amina/Imina X [GA]

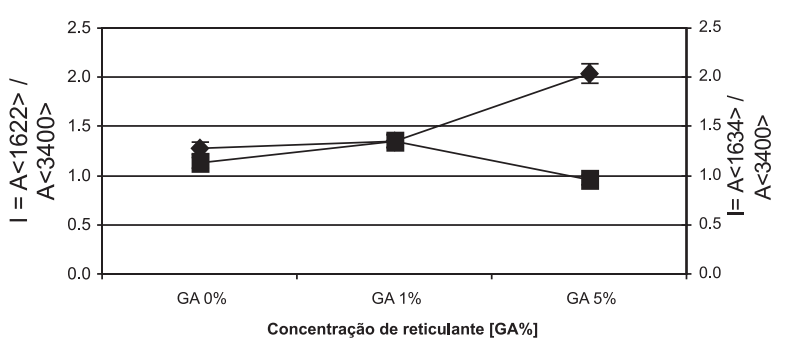

Figura 3. Evolução das bandas de formação de imina $(C=N)$ e redução de amina $\left(\mathrm{NH}_{2}\right)$, durante a reticulação da quitosana pelo $\mathrm{GA}$ na blenda $Q / P(1: 3)$

Reticulação do PVA

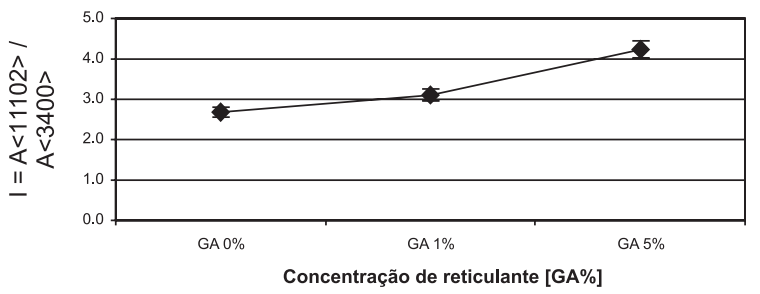

Figura 4. Evolução da banda de $1110 \mathrm{~cm}^{-1}$ durante a reticulação do PVA

\section{Titulação potenciométrica}

A partir da curva de titulação potenciométrica obtida a partir da solução de quitosana obtém-se a diferença de volume entre os pontos de inflexão que corresponde ao ácido consumido para a protonação dos grupos amina e permite a determinação do GD da quitosana. ${ }^{23}$ Após a correção do valor da concentração de $\mathrm{NaOH}$ obtém-se o valor de $76 \%$ para o GD..$^{24}$

\section{Difração de raios-X}

No difratograma da quitosana observaram-se dois picos, um de alta intensidade em $19,8^{\circ}$ e um de menor intensidade em $37,7^{\circ}$ que evidenciam a parte cristalina e uma ampla faixa abaixo dos picos, variando de aproximadamente 8 a $80^{\circ}$, onde predomina a forma amorfa do material. A cristalinidade calculada a partir da relação de áreas entre os picos e a área total sobre a curva do gráfico foi de aproximadamente $17 \%$. 
O difratograma do PVA GH=80\% apresentou três picos característicos de cristalinidade em $19,35^{\circ}$ (alta intensidade), $22,47^{\circ}$ (menos definido) e $40,28^{\circ}$ (menor intensidade) e uma ampla faixa abaixo dos picos, variando de aproximadamente $10 \mathrm{a} 75^{\circ}$, onde predomina a forma amorfa do material. A cristalinidade calculada semelhantemente ao descrito foi de $23 \%$.

No difratograma de filmes de quitosana pura e reticulados com GA, as áreas sobre os picos apresentaram uma relativa diminuição com o aumento do grau de reticulação. Os filmes de quitosana pura, reticulado com $1,0 \%$ e 5,0\%, apresentaram índices de cristalinidade de 23, 22 e 18\%, calculados a partir da relação de áreas, o que sugere que o aumento no nível de reticulação diminui o índice de cristalinidade dos filmes devido à diminuição dos graus de liberdade na conformação 3D.

\section{Propriedades mecânicas}

Todas as amostras foram preparadas com espessura de $250 \pm$ $150 \mu \mathrm{m}$ para assegurar uma seção transversal isenta de defeitos, a partir da mistura das soluções de quitosana e PVA nas proporções evidenciadas pela Tabela 1 e secas por evaporação do solvente após o ajuste do $\mathrm{pH}$ das soluções para 4,00 $\pm 0,05$. A Tabela 4 apresenta os valores de características geométricas médias das amostras, bem como os valores médios dos resultados obtidos nos ensaios de resistência mecânica à tração $(n>4)$.

O módulo de elasticidade é calculado a partir da inclinação da parte linear do gráfico e a deformação específica $(D E)$ corresponde a razão da variação de comprimento $(\Delta \mathrm{l})$ pelo comprimento inicial $\left(l_{0}\right)$ em porcentagem conforme a Equação 2 . A tenacidade corresponde à área sobre a curva até o colapso do CDP.

A Figura 5 apresenta os gráficos relativos às curvas de tensãodeformação no ensaio de tração para as amostras de filmes puros e blendas de PVA(a), Q/P(1:3)(b), Q/P(1:1) (c), Q/P(3:1) (d) e quitosana (e).

Observou-se que quanto maior o teor de quitosana na mistura implica no aumento da tensão máxima de tração, fato também observado por outros autores estudando misturas de QUI/PEO. ${ }^{25}$

Considerando o teor de reticulante, exceto para a blenda de $\mathrm{Q} / \mathrm{P}(1: 3)$, onde o aumento de [GA] $(0,1$ e $5 \%)$ não altera significativamente o valor da tensão máxima, como observado também

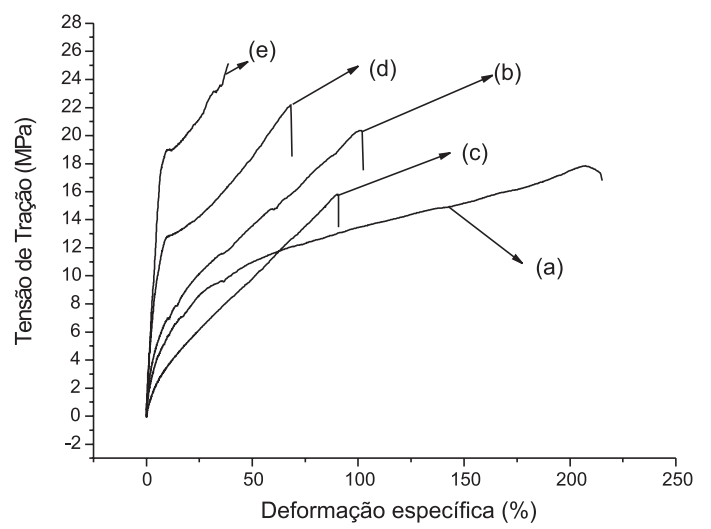

Figura 5. Gráfico de tensão $x$ deformação apresentando as curvas dos filmes sem reticulação de $P V A(a), Q / P(1: 3)(b), Q / P(1: 1)(c), Q / P(3: 1)(d) e$ quitosana(e)

no PVA puro; todas as demais blendas, inclusive a quitosana pura, sofreram uma redução na tensão máxima. Observou-se, ao contrário de Rokhade e Bahrami também analisando misturas de QUI/PVA, ${ }^{20,26}$ que a inclusão do reticulante GA reduziu a tensão máxima das misturas. Já Monteiro Jr. e Silva, ${ }^{21,27}$ analisando o efeito da reticulação da quitosana com o GA em amostras imersas em solução salina isotônica $(\mathrm{NaCl} 0,145 \mathrm{M}$ e $\mathrm{pH}=7,4 \pm 0,02)$ à temperatura ambiente e a $37^{\circ} \mathrm{C}$, observaram que o módulo de tração diminui quando pequenas quantidades de GA são usadas como reticulante e que o mesmo aumenta para concentrações de GA acima de $10 \%$, sugerindo ainda que a resistência mínima obtida para $1 \%$ de GA pode estar relacionada com o máximo equilíbrio alcançado para o grau de hidratação da amostra. Relatam também que resultados similares foram obtidos para amostras ensaiadas na condição de ambiente seco. Além disso, Berger, ${ }^{28}$ analisando hidrogéis de quitosana reticulados covalentemente, notou que a reação de reticulação pode induzir mudanças conformacionais da quitosana como observado por RMN quando reticulada com $1,1,3,3$, tetrametoxipropano e GA. Nesse caso, pode se inferir que na proporção de GA utilizado ocorre o aparecimento de tensões internas na estrutura das blendas, tornando-as mais frágeis.

Observou-se com relação entre a deformação específica e a concentração de quitosana a redução da deformação com o aumento do

Tabela 4. Características geométricas médias das amostras e valores de tensão, deformação, módulo de elasticidade e tenacidade com respectivos desvios padrão $(n>4)$

\begin{tabular}{|c|c|c|c|c|c|c|}
\hline $\mathrm{CP}$ & $\sigma_{\max }(\mathrm{MPa})$ & $\mathrm{DE}(\%)$ & $\mathrm{E}(\mathrm{MPa})$ & $\operatorname{Tn}(\mathrm{MJ} / \mathrm{m} 3)$ & esp. $(\mu \mathrm{m})$ & $\mathrm{A}\left(\mathrm{mm}^{2}\right)$ \\
\hline 1 & $20,0 \pm 2,9$ & $248 \pm 28,7$ & $74 \pm 15,7$ & $34,2 \pm 7,2$ & 300 & 0,83 \\
\hline 2 & $50,0 \pm 9,5$ & $45 \pm 5,1$ & $829 \pm 357,6$ & $16,8 \pm 3,0$ & 100 & 0,19 \\
\hline 3 & $17,0 \pm 1,6$ & $100 \pm 7,9$ & $97 \pm 28,3$ & $8,9 \pm 3,1$ & 200 & 0,60 \\
\hline 4 & $17,0 \pm 3,7$ & $100 \pm 7,9$ & $97 \pm 28,3$ & $8,9 \pm 3,1$ & 200 & 0,47 \\
\hline 5 & $25,0 \pm 5,1$ & $83 \pm 9,5$ & $442 \pm 131,0$ & $14,0 \pm 4,1$ & 200 & 0,52 \\
\hline 6 & $17,0 \pm 2,2$ & $269 \pm 28,0$ & $75 \pm 16,9$ & $30,5 \pm 5,4$ & 400 & 1,10 \\
\hline 7 & $34,0 \pm 2,2$ & $34 \pm 8,1$ & $1024 \pm 109,6$ & $8,4 \pm 3,8$ & 100 & 0,24 \\
\hline 8 & $13,0 \pm 2,6$ & $31 \pm 6,9$ & $149 \pm 15,0$ & $2,9 \pm 1,2$ & 200 & 0,42 \\
\hline 9 & $12,0 \pm 3,0$ & $48 \pm 12,4$ & $75 \pm 9,7$ & $2,4 \pm 1,7$ & 200 & 0,54 \\
\hline 10 & $22,0 \pm 4,4$ & $17 \pm 2,8$ & $634 \pm 215,1$ & $1,6 \pm 1,3$ & 100 & 0,31 \\
\hline 11 & $18,0 \pm 1,9$ & $254 \pm 19,8$ & $92 \pm 17,4$ & $27,4 \pm 4,3$ & 400 & 0,99 \\
\hline 12 & $28,0 \pm 2,4$ & $18 \pm 7,0$ & $913 \pm 238,9$ & $3,8 \pm 1,7$ & 100 & 0,36 \\
\hline 13 & $10,0 \pm 1,5$ & $31 \pm 8,6$ & $111 \pm 10,4$ & $1,9 \pm 0,7$ & 300 & 1,39 \\
\hline 14 & $13,0 \pm 2,4$ & $36 \pm 9,2$ & $210 \pm 60,2$ & $3,4 \pm 1,3$ & 300 & 1,14 \\
\hline 15 & $17,0 \pm 4,7$ & $6 \pm 3,2$ & $926 \pm 177,0$ & $1,4 \pm 1,3$ & 100 & 0,25 \\
\hline
\end{tabular}


teor de quitosana, ou seja, a blenda torna-se mais frágil. Em relação à deformação específica e o teor de GA nota-se que a incorporação de reticulante acentua a diminuição da deformação. O PVA puro apresenta um aumento de deformação específica para a [GA] de 1,0\% sofrendo uma diminuição para o aumento da $[\mathrm{GA}]$ para 5,0\%. Todas as blendas e a quitosana pura apresentaram uma diminuição relativa de deformação específica com o aumento de [GA]. Segundo Vachoud, ${ }^{29}$ analisando as propriedades físicas e fisico-químicas de hidrogéis de quitina relaciona o grau de acetilação (GDA) com o pH do meio durante a sinérese (processo de incorporação de fluido a partir da variação do $\mathrm{pH}$ do meio), evidenciando que para $\mathrm{pH}=4 \mathrm{o}$ valor do peso do gel no final da sinerese é maior e sugere que para este $\mathrm{pH}$, para um dado GDA, a densidade aparente de carga dos segmentos de quitina e a repulsão eletrostática são máximas. Os grupos amino livres presentes na cadeia do polímero estão em sua protonação máxima em um meio de relativamente baixa força iônica. Como conseqüência, a solvatação desses grupos iônicos está no máximo assim contribuindo para uma incorporação de fluido máxima. Quando o pH aumenta de 4 para 10 há uma desprotonação da função amino que é mais acentuada para menores GDA, devido à presença de um grande número de resíduos de glicosamina como é o caso da quitosana. Dessa observação podemos supor que a disponibilidade dos grupos amino da quitosana para reagirem com os aldeídos do GA é grande, favorecendo a um grau acentuado de reticulação e, conseqüentemente, deformando a estrutura interna aumentando as tensões no reticulado favorecendo a fragilização dos filmes.

Com relação ao módulo de elasticidade e à concentração de quitosana observou-se que o aumento do teor de quitosana na mistura aumenta o módulo de elasticidade da mesma, caracterizando um aumento na fragilidade, pois para uma mesma tensão a deformação diminui sensivelmente. Nesse aspecto notou-se ainda que com o aumento do teor de reticulante aumenta ainda mais o módulo acentuando a fragilidade. Para o PVA puro ocorre uma redução no módulo de elasticidade com o aumento da [GA], embora a deformação específica se mantenha entre 250 e $275 \%$. As blendas e a quitosana pura apresentaram uma variação máxima no módulo de elasticidade para a [GA] de 1,0\% sofrendo, praticamente, um retorno ao valor inicial na concentração de $5,0 \%$ de GA.

A Figura 6 apresenta a tenacidade (área sob a curva tensão $\mathrm{x}$ deformação até a fratura) observada nas blendas de QUI/PVA, bem como da QUI e PVA puros, em relação à variação do teor de quitosana. Observa-se que a tenacidade apresenta uma redução com o aumento do teor de quitosana até $40 \%$ e, a partir daí até $100 \%$, uma relativa recuperação. Observou-se ainda que a reticulação com o GA também reduz a tenacidade acentuadamente nas blendas e quitosana pura, entretanto para o PVA puro essa redução não se apresenta tão significativa.

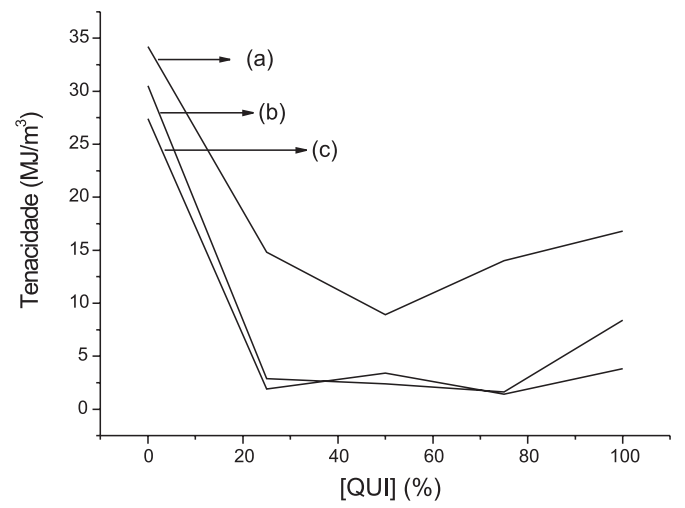

Figura 6. Influência da concentração de quitosana na tenacidade das blendas. Sem $G A(a), 1 \%$ de $G A(b)$ e $5 \%$ de $G A(c)$

\section{CONCLUSÕES}

Foram obtidos filmes de blendas de quitosana e PVA com e sem reticulação química visando aplicação em engenharia de tecido. Os filmes foram caracterizados por MEV, FTIR e XRD com o objetivo de evidenciar a morfologia, a presença de grupos químicos e a estrutura e arranjo cristalino, além de avaliar as propriedades mecânicas dos mesmos. Os resultados apresentam, além de indícios de falha de miscibilidade no filme da blenda $\mathrm{Q} / \mathrm{P}(1: 1)$, também forte evidência de que controlando o PVA e a quitosana, como precursores, associado a reticulação química é possível obter híbridos orgânico-orgânico, natural-sintético com variação das propriedades fisico-químicas em função da necessidade de aplicação.

\section{MATERIAL SUPLEMENTAR}

A Figura $1 \mathrm{~S}$, relativa à análise qualitativa dos filmes obtidos, as Figuras $2 \mathrm{~S}$ e $3 \mathrm{~S}$ referentes aos espectros de infravermelho e estruturas químicas da quitosana e PVA, respectivamente; a Figura 4S correspondente aos difratogramas da quitosana reticulada, as Figura $5 \mathrm{~S}$ e $6 \mathrm{~S}$ relativas às propriedades mecânicas de tração máxima em relação ao teor de quitosana e ao teor de GA dos filmes, respectivamente, encontram-se disponíveis em http://quimicanova.sbq.org.br, na forma de arquivo PDF, com acesso livre.

\section{AGRADECIMENTOS}

Ao suporte financeiro do CPGEM/UFMG, CAPES e FAPEMIG/ Brasil. E. S. Costa Jr. também agradece ao CEFET-MG as horas investidas neste trabalho.

\section{REFERÊNCIAS}

1. Drury, J. L.; Mooney, D. J.; Biomaterials 2003, 24, 4337.

2. Berger, J.; Reist, M.; Mayer, J. M.; Felt, O.; Gurny, R.; Euro. J. Pharm. Biopharm. 2004, 57, 35.

3. Suh, J. K. F.; Matthew, H. W. T.; Biomaterials 2000, 21, 2589.

4. Mansur, A. A. P.; Mansur H. S.; Anais do $8^{\circ}$ Congresso Brasileiro de Polímeros, Águas de Lindóia, Brasil, 2005.

5. Lin, W.; Yu, D.; Yang, M.; Colloids Surf. B 2006, 47, 43.

6. Orefice, R. L. Em Biomateriais: Fundamentos \&Aplicações; Oréfice, R. L.; Pereira, M. M.; Mansur, H. S., eds.; Cultura Médica: Rio de Janeiro, 2006, cap. 4.

7. Rokhade, A. P.; Patil, S. A.; Aminabhavi, T. M.; Carbohydr. Polym. 2007, 67, 605 .

8. Beppu, M. M.; Arruda, E. J.; Santana, C. C.; Polímeros: Ciência e Tecnologia 1999, 4, 163.

9. Bunn, C. W.; Nature 1948, 4102, 929

10. Cho, J. D.; Lyoo, W. S.; Chvalun, S. N.; Blackweel, J.; Macromolecules 1999, 32, 6236

11. Ricciardi, R.; Gaillet, C.; Ducouret, G.; Lafuma, F.; Lauprêtre, F.; Polymer 2003, 44, 3375.

12. Clark, G. L.; Smith, A. F.; J. Phys. Chem. 1936, 40, 863.

13. Mazeau, K.; Winter, W. T.; Chanzy, H.; Macromolecules 1994, 27, 7606.

14. Ogawa, K.; Agric. Biol. Chem. 1991, 55, 2375.

15. ASTM - American Standard Testing and Material; ASTM D882/02: Standard Test Method for Tensile Properties of Thin Plastic Sheeting, New York, 2002.

16. Don, T. M.; King, C. F.; Chiu, W. Y.; Peng, C. A.; Carbohydr. Polym. 2006, 63, 331.

17. Brugnerotto, J.; Lizardi, J.; Goycoolea, F. M.; Argüelles-Monal, W.; 
Desbrières, J.; Rinaudo, M.; Polymer 2001, 42, 3569.

18. Wang, T.; Turhan, M.; Gunasekaran, S.; Polym. Int. 2004, 53, 911.

19. Rao, P. S.; Sumitha, B.; Sridhar, S.; Krishnaiah, A.; Sep. Purif. Technol. 2006, 48, 244

20. Rokhade, A. P.; Shelke, N. B.; Patil, S. A.; Aminabhavi, T. M.; Carbohydr. Polym. 2007, 69, 678.

21. Monteiro Jr., O. A. C.; Airoldi, C.; Int. J. Biol. Macromol. 1999, 26, 119.

22. Costa Jr., E. S.; Mansur, H.; Anais 11 International Macromolecular Colloquium, Gramado, Brasil, 2007.

23. Tolaimate, A.; Desbrères, J.; Rhazi, M; Alagui, A.; Vincendon, M.; Vottero, P.; Polymer 2000, 41, 2463.
24. Tan, S. C.; Khor, E.; Tan, T. K.; Wong, S. M.; Talanta 1998, 45, 713.

25. Zivanovic, S.; Li, J.; Davidson, P. M.; Kit, K.; Biomacromolecules 2007, $8,1505$.

26. Bahrami, S. B.; Kordestani, S. S.; Mirzadeh, H.; Mansoori, P.; Iranian Polymer J. 2003, 12, 139.

27. Silva, R. M.; Silva, G. A.; Coutinho, O. P.; Mano, J. F.; Reis, R. L.; J. Mater Sci.: Mater. Med. 2004, 15, 1105.

28. Berger, J.; Reist, M.; Mayer, J. M.; Felt, O.; Peppas, N. A.; Gurny, R.; Euro. J. Pharm. Biopharm. 2004, 57, 19.

29. Vachoud, L.; Domard, A.; Biomacromolecules 2001, 2, 1294. 


\section{PREPARAÇÃO E CARACTERIZAÇÃO DE BLENDAS DE QUITOSANA/POLI(ÁLCOOL VINÍLICO) RETICULADAS QUIMICAMENTE COM GLUTARALDEÍDO PARA APLICAÇÃO EM ENGENHARIA DE TECIDO}

\section{Ezequiel de Souza Costa Jr.}

Coordenação do Curso Técnico de Mecânica, Centro Federal de Educação Tecnológica - MG, Av. Amazonas, 5253,

30480-000 Belo Horizonte - MG, Brasil

Herman Sander Mansur*

Departamento de Engenharia Metalúrgica e de Materiais, Universidade Federal de Minas Gerais, Rua Espírito Santo, 35, 30160-030 Belo Horizonte - MG, Brasil
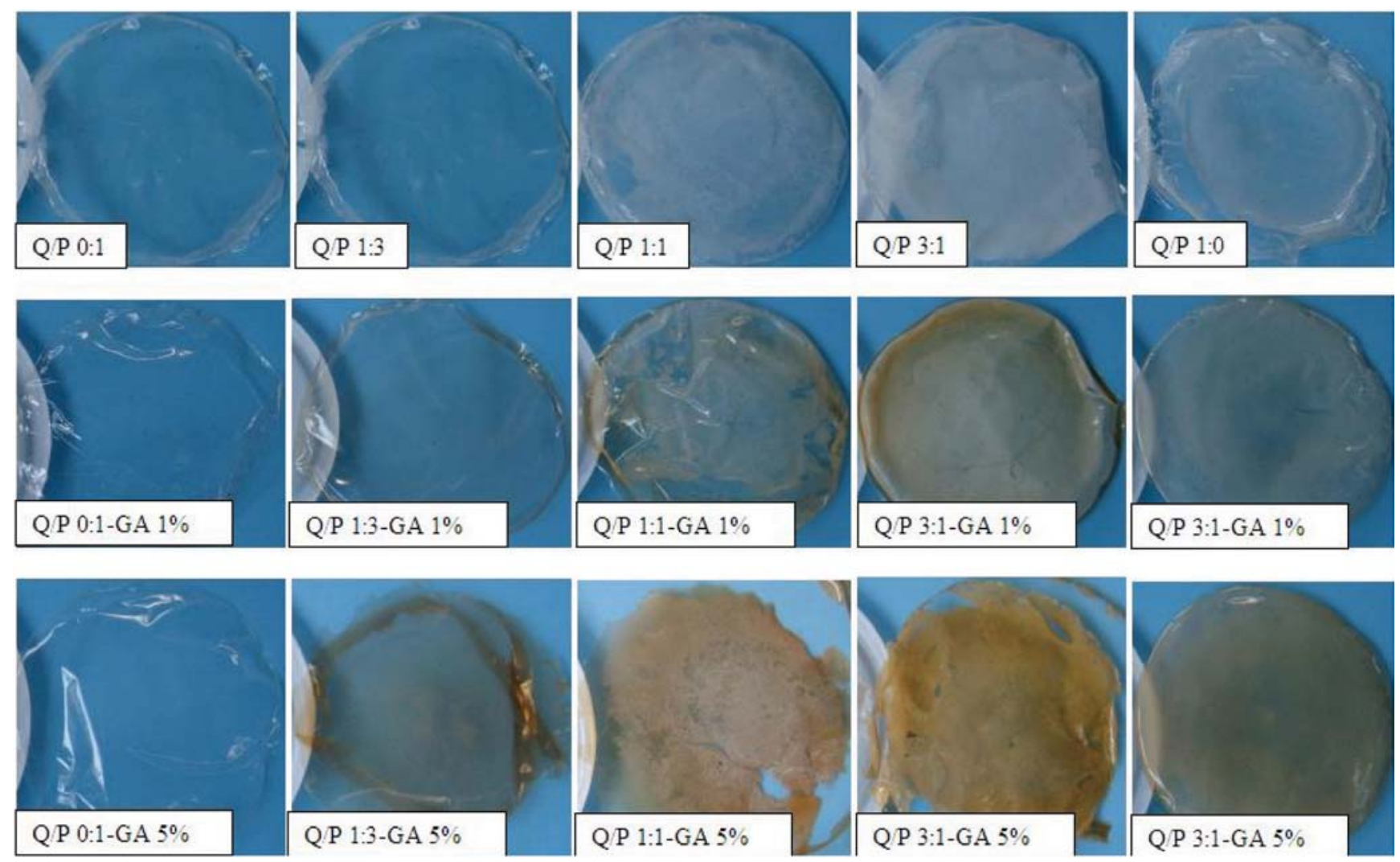

Figura 1S. Filmes das blendas de QUI/PVA, quitosana e PVA puros e reticulados com $1 \%$ e $5 \%$ de GA 


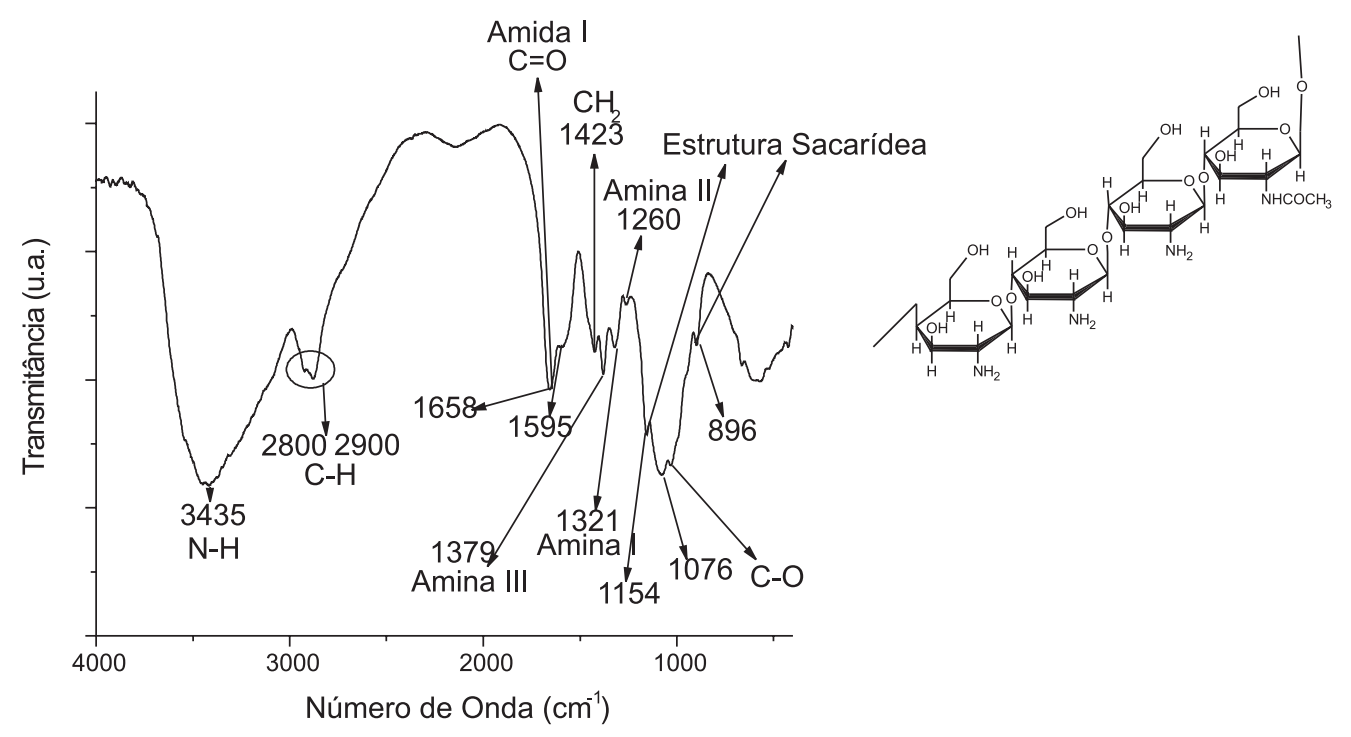

Figura 2S. Espectro de IV típico da quitosana $(G D=75,6 \%)$ e estrutura química da quitosana parcialmente desacetilada

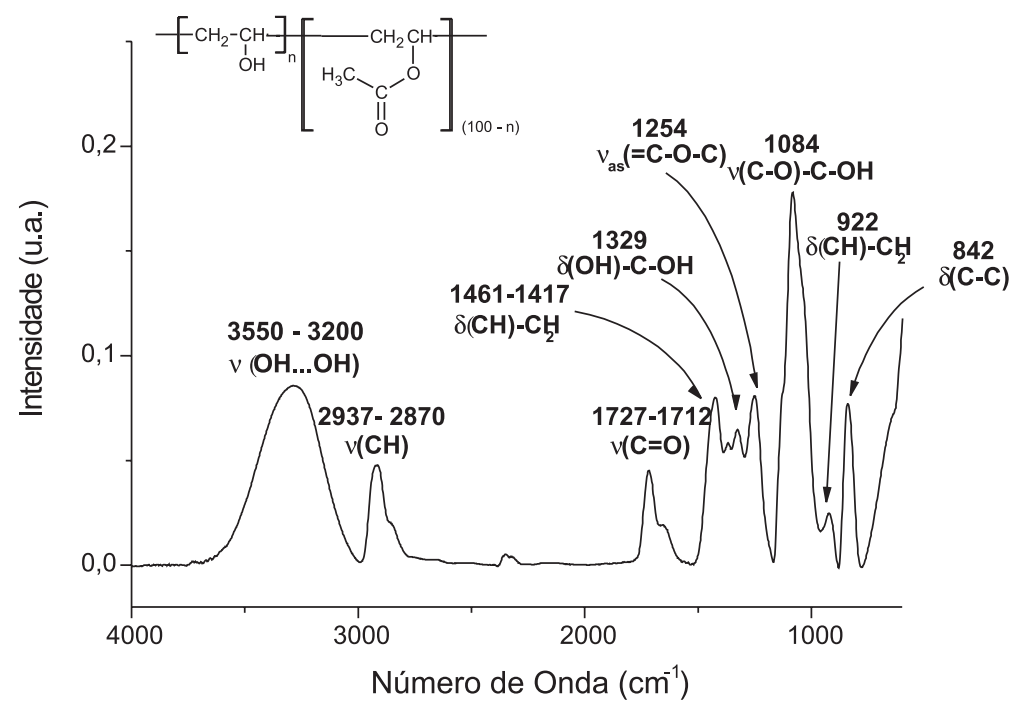

Figura 3S. Espectro de IV típico do PVA e estrutura química do PVA parcialmente hidrolisado (GH=80\%) 


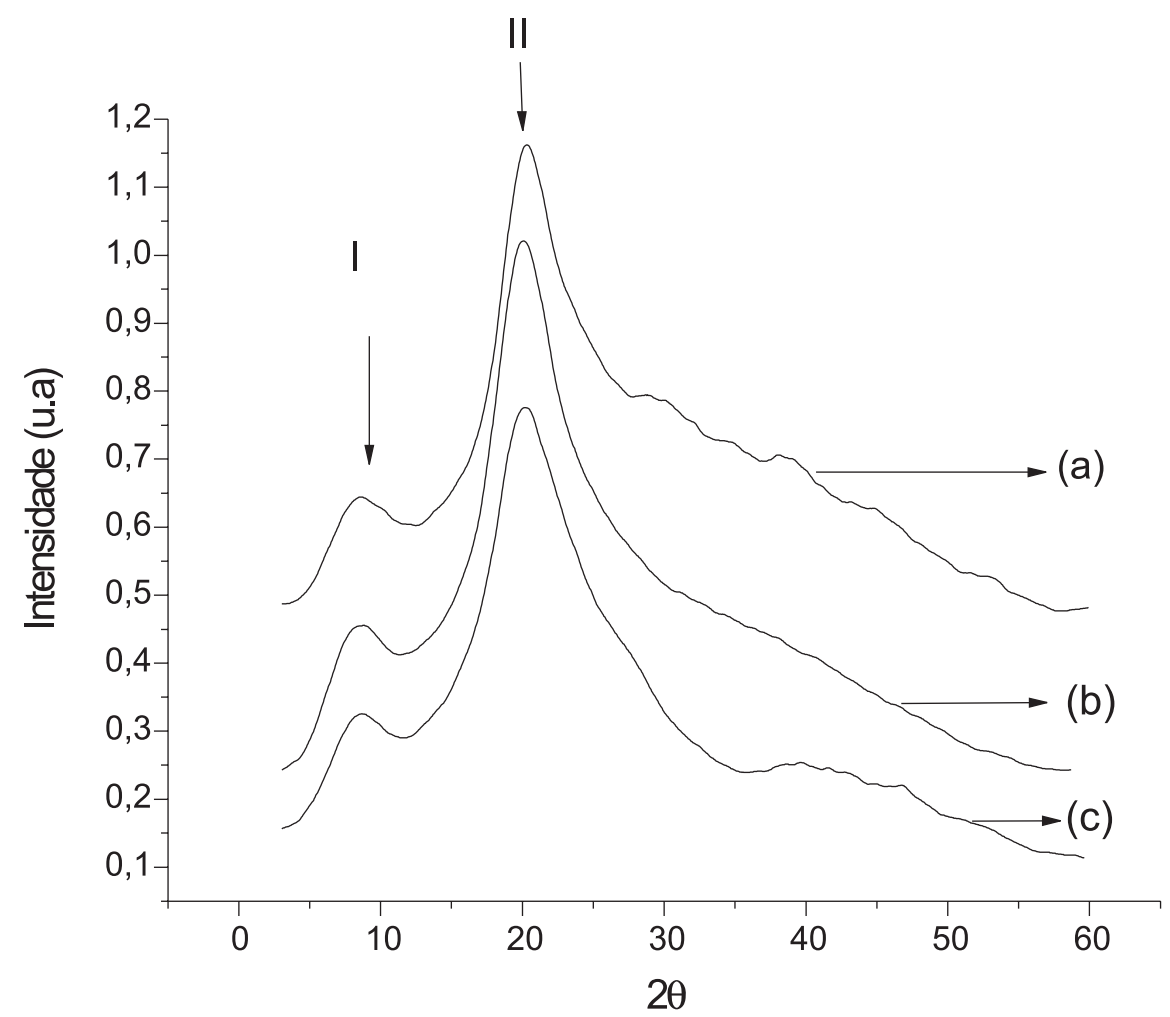

Figura 4S. Difratogramas da quitosana pura e com [GA] de 1,0\% e 5,0\%, (a), (b) e (c)

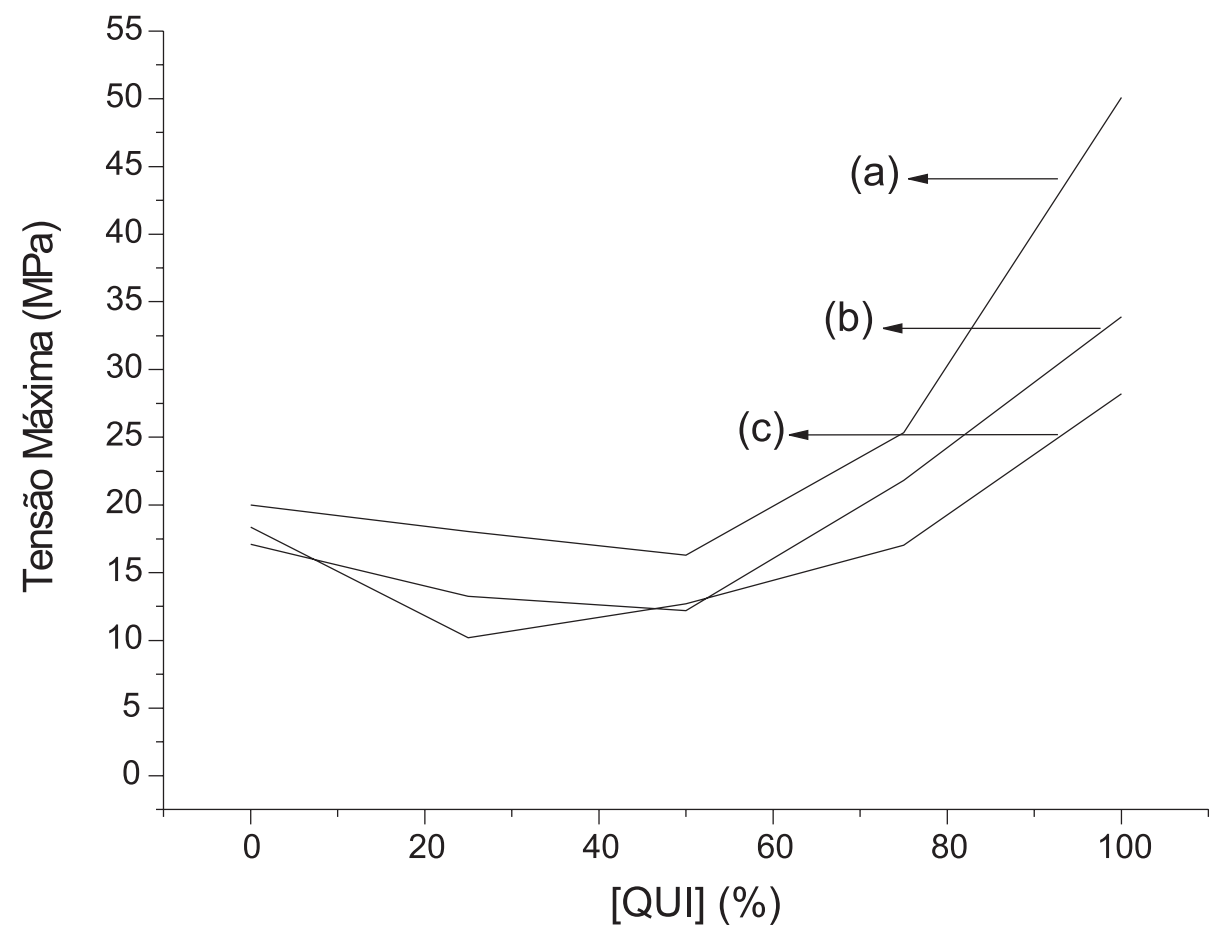

Figura 5S. Influência da concentração de quitosana na tensão máxima de tração. Sem $G A(a), 1 \%$ de $G A(b)$ e $5 \%$ de $G A(c)$ 
Tensão Máxima x [GA]

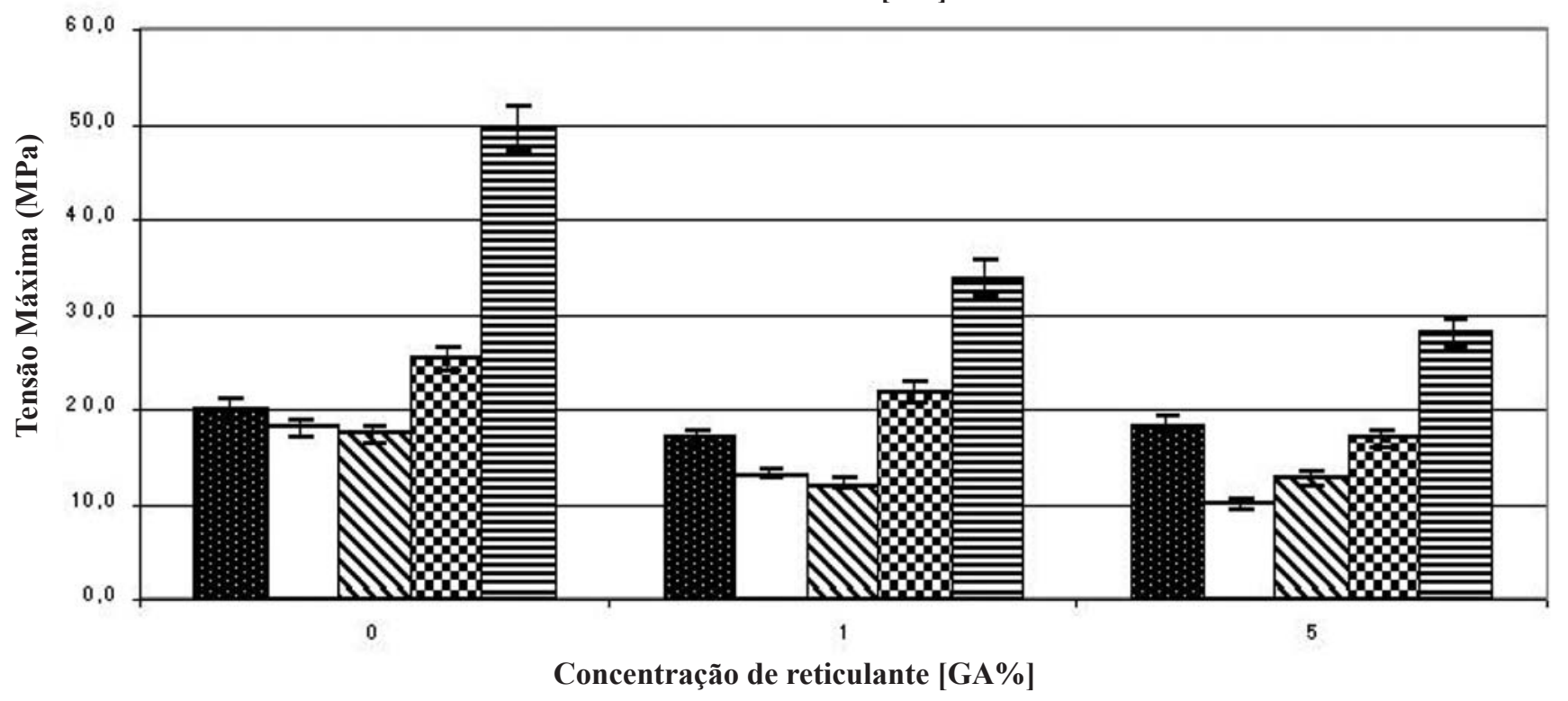

Figura 6S. Influência da concentração de GA na tensão máxima de tração. $\mathrm{PVA} \mathbf{\mathbf { X }}, \mathrm{Q} / \mathrm{P}(1: 3) \sqsubset, \mathrm{Q} / \mathrm{P}(1: 1) \mathbf{N}, \mathrm{Q} / \mathrm{P}(3: 1) \mathbf{X}, \mathrm{QUI} \equiv$ 